\title{
The diffusion process of metastable carriers in bismuth
}

\author{
J. C. G. de Sande, M. Sánchez Balmaseda, ${ }^{\text {a) }}$ and J. M. Guerra Pérez \\ Departamento de Optica, Facultad de Ciencias Físicas, Universidad Complutense, 28040 Madrid, Spain
}

\begin{abstract}
The diffusion process of electrons and holes which have been pumped into a metastable band in bismuth films by a $1.064-\mu \mathrm{m}$ laser pulse is considered in the calculation of the induced thermal gradient. The fit between the temporal evolution of this calculated thermal gradient and that of the thermoelectric response of films to the laser excitation allows us to estimate an upper limit of the ambipolar diffusion coefficient of metastable carriers.
\end{abstract}

\section{INTRODUCTION}

It is well known that pulsed laser irradiation generates transverse thermoelectric potentials in metallic films. The origin of these potentials is believed to be the in-grown tensions produced by controlled or uncontrolled asymmetries in the deposition process or some kind of texturation in the film plane. $^{1-4}$

The heat diffusion equation has been often used to calculate the thermal distribution produced by laser pulses in surfaces ${ }^{5}$ and in thin films. ${ }^{6}$ When the laser pulses temporal width is in the nanosecond-time scale and the radiation intensity is moderate, it has been experimentally shown that the heat diffusion equation predicts the temporal evolution of the induced temperature increase of a metallic surface..$^{7-9}$

We have previously studied the potentials induced in bismuth films by $1.064-\mu \mathrm{m}$ laser pulses with and without an applied magnetic field along the film. The anomalous temporal evolution of the thermomagnetic and the thermoelectric responses then observed was well explained by assuming that electrons were optically pumped into a metastable band and that remained frozen in the position where they were excited. With this hypothesis, very good agreement between experimental results and theoretical calculations was obtained..$^{10,11}$ However, we have recently reported a weak laser-induced transient photoconductivity in bismuth films. ${ }^{12}$ This observation means that the excited carriers have a measurable mobility. Thus, being the carriers inhomogeneously excited, it is foreseeable a spatial diffusion of these carriers during the time scale of the phenomenon.

Here we report a theoretical study of the temporal evolution of the thermal gradient induced in the film considering that an ambipolar diffusion of excited carriers is taking place. The fitness to the above mentioned experimental thermoelectric response allow us to give an estimation of the upper limit of the ambipolar diffusion coefficient of the pumped carriers in the metastable band.

\section{THEORETICAL APPROACH}

As we have mentioned above, irradiation of bismuth with $1.17 \mathrm{eV}$ photons produces a pumping of electrons from the valence band to superior metastable states. In bismuth,

\footnotetext{
alDepartamento de Física Aplicada III (Electricidad y Electrónica).
}

the $T_{6}^{+}$level is some tenths of $\mathrm{eV}$ above the Fermi level..$^{13-16}$ Then the optically pumped electrons may be excited to this level after a fast nonradiative decay, having these carriers, in general, different properties from those of electrons remaining in the conduction band (see Fig. 1).

When there are carriers of different sign and their densities are $\geqslant 10^{14} \mathrm{~m}^{-3}$, Coulombian forces greatly influence the carriers spread process and ambipolar diffusion has to be considered. ${ }^{17}$ The mean electrons and holes densities pumped by a laser pulse can be roughly estimated by $A w_{r} / d h \nu_{L}$, where $A$ is the material absorption coefficient, $w_{r}$ is the ratio $E_{r} / s$, being $E_{r}$ the laser pulse energy and $s$ the beam cross section at the film position, $d$ is the film thickness, and $h \nu_{L}$ is the photons energy. Under our experimental conditions $\left(w_{r} \geqslant 10 \mathrm{~J} / \mathrm{m}^{2}, d \simeq 5.5 \mu \mathrm{m}\right.$, and $h \nu_{L} \simeq 1.17 \mathrm{eV}$ ), densities bigger than $10^{23} \mathrm{~m}^{-3}$ are obtained. Then, if we denote by $n(x, t)$ the density of pumped pairs at a given distance $x$ from the film surface and at a given instant $t$, these excited carriers in the irradiated bismuth film must obey the following diffusion equation:

$$
\frac{\partial n(x, t)}{\partial t}=\frac{\phi(x, t)}{h \nu_{L}}+D_{a} \frac{\partial^{2} n(x, t)}{\partial x^{2}}-S_{m} n(x, t),
$$

where $D_{i t}$ is the ambipolar diffusion coefficient, $S_{m}$ is the rate of recombination processes, and $\phi(x, t)$ is the thermal power per unit of volume entering the film that will be given by

$$
\phi(x, t)=\phi_{0} \exp (-\delta x / d) f(t),
$$

being

$$
\phi_{0}=\frac{A w_{r}}{\int_{0}^{d} \exp (-\delta x / d) d x \int_{0}^{\infty} f\left(t^{\prime}\right) d t^{\prime}},
$$

where $\delta=d / x_{L}, x_{L}$ is the attenuation distance of the laser radiation in the material and $f(t)$ is the temporal profile of the laser pulse. Then the term $\phi(x, t) / h \nu_{L}$ represents the number of excited pairs produced by the laser pulse per unit of volume and time if a quantum efficiency equal to unity is assumed.

The recombination rate $S_{m}$ has been previously estimated assuming the hypothesis of frozen carriers. ${ }^{10,11} \mathrm{Nev}$ ertheless, $D_{a}$ is completely unknown. This coefficient, in principle, has to be low since the frozen carriers hypothesis 


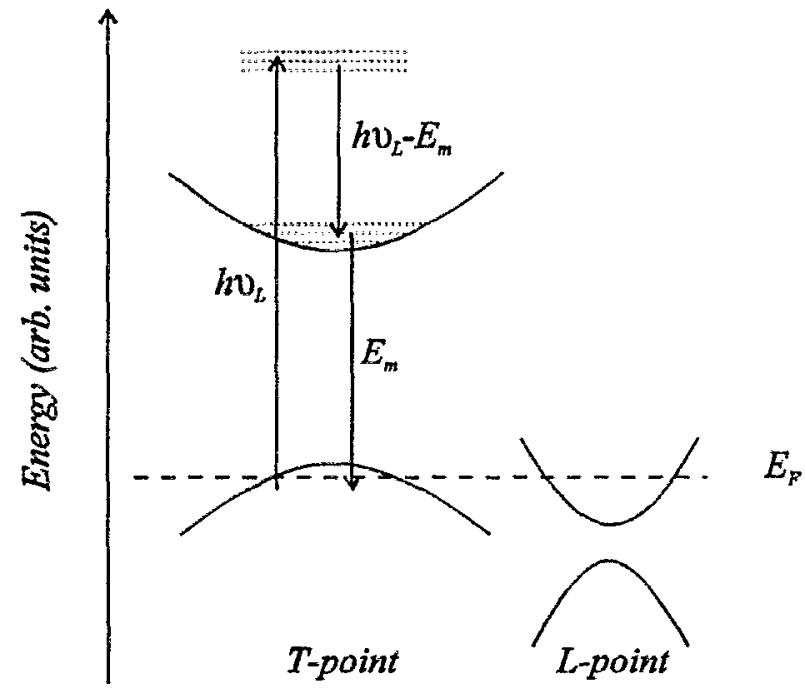

FG. 1 . Sehematic of the $L$-point and $T$-point band alignments in bismuth.

accounted for the results quite well. In order to estimate an upper limit of this coefficient, we have studied the temporal evolution of the transverse thermoelectric response to a $1.064-\mu \mathrm{m}$ laser pulse. Under our experimental conditions, we have previously shown that the generated thermoelectric potential is independent of the thermal distribution across the film and only depends on the temperature of each surface of the film in the following way:

$$
V_{t}(t)=l \beta_{z x} \frac{T(d, t)-T(0, t)}{d}
$$

where $l$ is the distance between electrodes, $T$ denotes ternperature, and $\beta_{z x}$ is the transverse thermopower coefficient. ${ }^{11}$

The evolution of the film temperature distribution $T(x, t)$ is the solution of the heat diffusion equation:

$$
\rho^{*} c_{p} \frac{\partial T(x, t)}{\partial t}=\phi_{s}(x, t)+K_{t} \frac{\partial^{2} T(x, t)}{\partial x^{2}}
$$

where $\rho^{*}$ is the mass density, $c_{p}$ is the heat capacity, $K_{t}$ is the thermat conductivity, and $\phi_{s}(x, t)$ is the source term. The laser power is mainly absorbed through interband transitions and the excited electrons transmit part of their energy to the lattice in a quick nonradiative relaxation process occupying the levels that are closest to the band edge. The rest of the energy is liberated in a recombination process that takes a time of about some tens of nanoseconds. With this model, the thermal power per unit of volume transferred to the lattice is

$$
\begin{aligned}
\phi_{s}(x, t)= & \left(1-\frac{E_{m}}{h \nu_{L}}\right) \phi_{0} \exp (-\delta x / d) f(t) \\
& +E_{m} S_{m} n(x, t)
\end{aligned}
$$

where $E_{m}$ is the average recombination energy. ${ }^{10,11}$

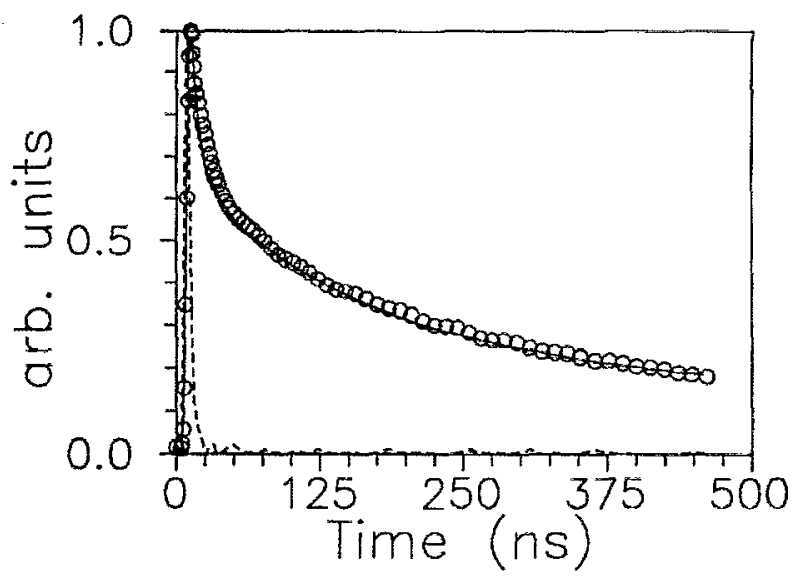

FIG. 2. Experimental (circles) and theoretical (solid line) transverse thermoelectric response of a $5.5-\mu \mathrm{m} \mathrm{Bi}$ film to a $1.064-\mu \mathrm{m}$ laser pulse (dashed line). The theoretical curve is obtained with $E_{m}=0.64 \mathrm{eV}, S_{m}^{-1}=57 \mathrm{~ns}$, and $D_{a}=1.5 \times 10^{-7} \mathrm{~m}^{2} / \mathrm{s}$.

\section{RESULTS AND DISCUSSION}

In order to calculate the source term of the heat diffusion equation given by Eq. 6 , it is necessary to obtain $n(x, t)$ from Eq. (1) where an ambipolar diffusion of the pumped carriers has been considered. Equations (1) and (5) have been solved by a standard numerical method. ${ }^{18}$ The temporal evolution of $T(d, t)-T(0, t)$ thus obtained has been plotted together with the experimental thermoelectric response in Fig. 2. The experimental thermoelectric signal corresponds to an average of ten registers. The film used in the experiment had a thickness of $d \simeq 5.5-\mu \mathrm{m}$ and it was grown on a glass substrate by vacuum evaporation ( $10^{-6}$ Torr) of $99.9999 \%$-pure bismuth at room temperature. The film was irradiated with a Q-switched and polarized Nd-YAG laser (wavelength $\approx 1.064 \mu \mathrm{m}$ ), having a typical temporal pulse width of $\approx 6$ ns (see Fig. 2). The irradiation energy density per pulse was $w_{r}=33 \mathrm{~J} / \mathrm{m}^{2}$. For calculations, the bismuth physical constants $A=0.33, c_{p}=125.4 \mathrm{~J} / \mathrm{kg} \mathrm{K}, \rho^{*}=9747 \mathrm{~kg} / \mathrm{m}^{3}$, $K_{t}=6.0 \mathrm{~W} / \mathrm{mK}$, and $x_{L}=20 \mathrm{~nm}$ were taken from current literature. ${ }^{19,20}$ In order to evaluate the goodness of fit of the model to the observed data, we used the square of the correlation coefficient. Excellent fits as the one shown in Fig. 2, having square correlation coefficients bigger than 0.99 , are obtained with the following values: $0.62 \mathrm{eV}<E_{m}<0.68 \mathrm{eV}$, $45 \mathrm{~ns}<S_{m}^{-1}<65 \mathrm{~ns}$, and $D_{a}<4 \times 10^{-7} \mathrm{~m}^{2} / \mathrm{s}$.

The values obtained for the average recombination energy and for the recombination rate are in full agreement with the ones already obtained. From the study of the photoconductive effect in bismuth, we obtained an estimation of the sum of the diffusion coefficients of the pumped electrons and holes: $D_{e}+D_{h} \sim 4 \times 10^{-6} \mathrm{~m}^{2} / \mathrm{s} .{ }^{12}$ Such a value is somewhat higher than the upper limit of the ambipolar diffusion coefficient found in the present work; then, the pumped electrons (or the pumped holes) are quite slower than the pumped holes (or the pumped electrons). The pumped electrons are generated in the $T_{0}^{+}$metastable band that could be quite different from the conduction band. ${ }^{12}$ It is not clear which is the concrete band where the excited holes lay. The 
ambipolar diffusion coefficient can be expressed as a func tion of the individual diffusion coefficients: ${ }^{17}$

$$
D_{a}=\frac{D_{e} / \mu_{e}+D_{h} / \mu_{h}}{1 / \mu_{e}+1 / \mu_{h}}
$$

where $\mu$ is the carriers mobility, and the subscripts $e$ and $h$ refer to electrons and holes respectively. Using the Einstein relation, $D_{j} / \mu_{j}=k_{B} T / e$ where $j$ denotes the kind of carriers, $k_{B}$ is the Boltzmann constant, and $e$ is the electron charge, the diffusion coefficient of the slowest metastable carriers $D_{s}$ is bounded by

$$
D_{s}=D_{a} / 2 \leqslant 2 \times 10^{-7} \mathrm{~m}^{2} / \mathrm{s} \text {. }
$$

On the other hand, the diffusion coefficient of the fastest metastable carriers $D_{f}$ can be estimated as:

$$
D_{f}=D_{h}+D_{e} \sim 4 \times 10^{-6} \mathrm{~m}^{2} / \mathrm{s} \text {. }
$$

Unfortunately, the available experimental data do not allow us to identify which carriers diffuse slowly and which ones diffuse quickly.

At room temperature, the mobilities of the electrons (in the conduction band) and holes (in the valence band) are in the ranges $0.1-1 \mathrm{~m}^{2} / \mathrm{V} \mathrm{s}$ and $0.02-0.2 \mathrm{~m}^{2} / \mathrm{V} \mathrm{s}$, respectively, for films thicknesses below $1 \mu \mathrm{m} .^{21-23}$ In the case of bulk samples, the electrons mobilities are in the $0.5-2 \mathrm{~m}^{2} / \mathrm{V} \mathrm{s}$ range, and the holes mobilities in the $0.2-1 \mathrm{~m}^{2} / \mathrm{Vs}$ range. ${ }^{24-26}$ Making use again of the Einstein relation, the above mentioned mobilities multiplied by $k_{B} T / e=0.026 \mathrm{~V}$ give the values of those carriers diffusion coefficients. It must be noticed that the ambipolar diffusion coefficient for pumped carriers is several orders of magnitude lower than any of the diffusion coefficients of the nonoptically excited electrons and holes. This fact means that the metastable carriers have very different transport properties from those of the thermal carriers.
' R. J, von Gutfeld, Appl. Phys. Lett. 23, 206 (1973).

${ }^{2}$ V. I. Andreev, A. B. Granovskii, and V. A. Yakolev, Kvant. Electron. (Moscow) 15, 1295 (1985) [translation: Sov. J. Quantum Electron. 15, 857 (1985)].

${ }^{3}$ G. A. Ukhlinov, Z. Y. Kosakovskaya, and V. N. Vigdorovich, Inorg. Mater. 22, 827 (1986).

${ }^{4}$ S. M. Javed Akhtar and D. Ristau, Phys. Status Solidi A 109, 255 (1988).

${ }^{5}$ J. H. Bechtel, J. Appl. Phys. 46, 1585 (1975).

${ }^{6}$ F. Bloisi and L. Vicari, Appl. Phys. B 47, 67 (1988).

${ }^{7}$ J. M. Hicks, L., E. Urbach, E. W. Plummer, and H. L. Dai, Phys. Rev. Lett. 61, 2588 (1988).

${ }^{8}$ H. E. Elsayed-Alì and J. W. Herman, Appl. Phys. Lett. 57, 1508 (1990).

${ }^{9}$ S. S. Mam, B. D. Todd, J. T. Stuckless, T. Seto, and D. A. King, Chem. Phys. Lett. 183, 529 (1991).

${ }^{10} \mathrm{M}$. Sánchez Baimaseda and J. M. Guerra, Phys. Rev. B 41, 10372 (1990).

${ }^{11}$ J. C. G. de Sande, M. Sánchez Balmaseda, and J. M. Guerra Pérez, Phys. Rev, B 47, 9844 (1993).

${ }^{12}$ J. C. G. de Sande, M. Sánchez Balmaseda, and J. M. Guerra Pérez, Phys. Rev. B 50, 1861 (1994).

${ }^{13}$ X. Gonze, J. P. Michenaud, and J. P. Vigneron, Phys. Rev, B 41, 11827 (1990).

14V. De Renzi, M. Grazia Betti, and C. Mariani, Phys. Rev. B 48, 4767 (1993).

${ }^{15}$ J. P. Omagio, J. R. Meyer, C. A. Hoffman, A. DiVenere, X. J. Yi, C. L. Hou, H. C. Wang, J. B. Ketterson, G, K. Wong, and J. P. Heremans, Phys. Rev. B 48, 11439 (1993).

${ }^{16}$ J. H. Xu, E. G. Wang, C. S. Ting, and W. P. Su, Phys. Rev. B 48, 17271 (1993).

${ }^{17}$ E. W. McDaniel and E. A. Mason, The Mobility and Diffusion of Ions in Gases (John Wiley \& Sons, New York, 1973).

${ }^{18}$ W. Kreith, Basic Heat Transfer (Harper \& Row, New York, 1980).

${ }^{19}$ AIP Handbook, 3rd ed, edited by Dwitght E. Gray (AIP, New York, 1972).

${ }^{20}$ Handbook of Chemistry and Physics, 53 rd ed., edited by Robert C. Weast (Chemical Rubber Co., Cleveland, OH, 1972-73).

${ }^{21} \mathrm{~S}$. Kochowski and A. Opilski, Thin Solid Films 48, 345 (1978).

${ }^{22}$ L. K. J. Vandamme and J, Kedzia, Thin Solid Films 65, 283 (1980).

${ }^{23}$ U. Dillner and W. Schnelle, Phys. Status Solidi A 116, 715 (1989).

${ }^{24}$ C. F. Gallo, B. S. Chandrasekar, and P. H. Sutter, J. Appl. Phys. 34, 144 (1963).

${ }^{25}$ O. P. Hansen, E. Cheruvier, J. P. Michenaud, and J. P. Issi, J. Phys. C 11, 1825 (1978).

${ }^{26}$ T. F. I. Mikhail, O. P. Hansen, and H. Nielsen, J. Phys. C 13, 1697 (1980). 